\title{
Loss of STARD7 Expression Compromises Epithelial Barrier Integrity in Airway of the $\mathrm{Shh}^{+/} \mathrm{Cre} / \mathrm{STARD7}{ }^{-/}$Mice
}

\author{
C.-L. Na ${ }^{1}$, L. Yang ${ }^{1}$ and T.E. Weaver ${ }^{1}$ \\ ${ }^{1 .}$ Cincinnati Children's Hospital Medical Center, Division of Pulmonary Biology, Cincinnati, OH, USA
}

The steroidogenic acute regulatory protein-related lipid transfer (START) domain containing proteins are family of 15 proteins that regulate various biological functions including lipid transport, metabolism, and signaling. Our studies by immune blotting and immunocytochemistry showed that STARD7, one of the START proteins, is expressed in lung epithelial cells. Loss of single allele of STARD7 in global KO mice $\left(\mathrm{STARD}^{+/-}\right.$) is sufficient to induce airway hyperactivity to ovalbumin challenge, increased epithelial barrier permeability, and atopic dermatitis. It is not clear if hyperactivity to ovalbumin is directly related to loss of STARD7 functions in airway epithelial cells or if other cells contribute to this phenotype. In this study, we generated mice in which expression of STARD7 was selectively disrupted in lung epithelial cells. The loss of STARD7 expression compromised epithelial barrier integrity, providing further evidence that STARD7 is critical for maintaining airway cell homeostasis.

$\mathrm{Shh}^{+/} \mathrm{Cre} / \mathrm{STARD} 7^{-/-}$mice (STARD7 KO mice) were generated for selective disruption of STARD7 expression in lung epithelial cells. SEM studies using mouse lungs obtained from 4 month and 10 month old STARD7 KO mice (Figure 1) determined that they had shorter and fewer cilia and microvilli in small bronchi and bronchioles compared to age-matched controls. Fractures along luminal cell surface were detected in small bronchi and bronchioles and were more pronounced in 10 month old KO mice than in 4 month old $\mathrm{KO}$ mice.

To determine if epithelial cell structure was disrupted in airway of the STARD7 KO mice, lung blocks of age-matched WT and KO mice were collected for TEM analysis. There was a significant reduction in the number and size of mitochondria in ciliated bronchiolar epithelial cells and Clara cells of the 4 month and 10 month old STARD7 KO mice (Figure 2). The decrease in mitochondrial population was often associated with formation of mitochondrial limiting membrane bound lipid inclusions, disintegration and degradation of mitochondrial cristae and matrix, suggesting that STARD7 is important for maintaining lipid homeostasis in mitochondria.

In distal airway of the 4 and 10 month old STARD7 KO mice, perinuclear lipid inclusions were detected in ciliated bronchiolar epithelial cells and Clara cells. Concentric lamellar inclusions with or without degraded mitochondria and other cellular fragments were prominent in some Clara cells. Ciliated bronchiolar epithelial cells and Clara cells that had lipid accumulation and lipid inclusions often had leaky basolateral cell junctions and loose intercellular spaces, suggested that integrity of airway epithelial barrier was compromised.

Taken together, our studies demonstrate that loss of STARD7 function in bronchiolar epithelial cells results in aberrant mitochondrial formation and compromised integrity in airway epithelial barrier. How STARD7 regulates mitochondria and epithelial barrier integrity in airway cells under asthmatic challenge will be determined in future studies. 

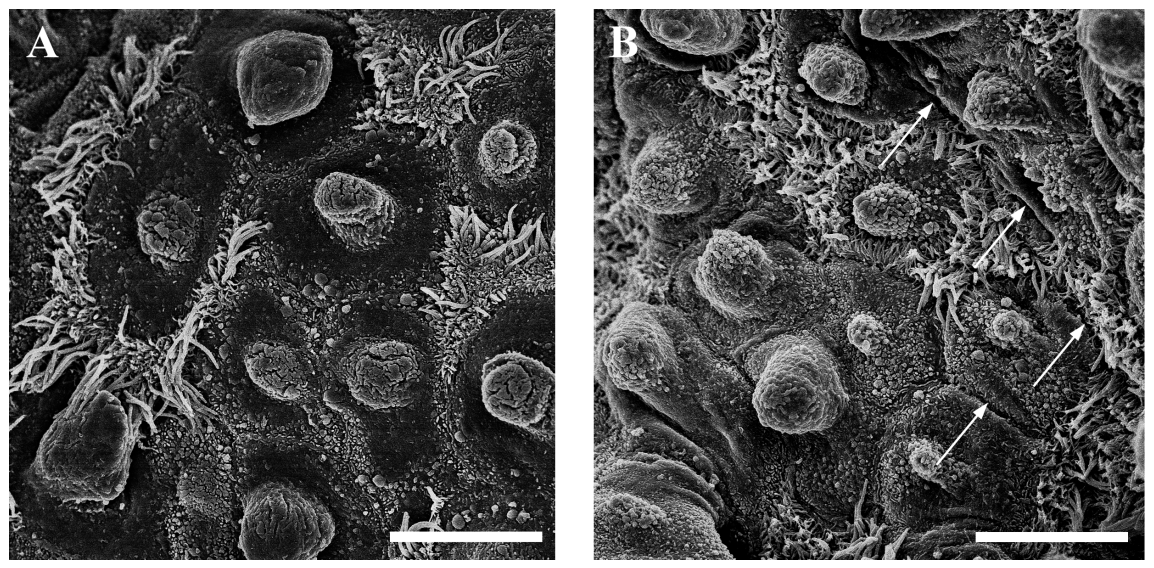

Figure 1. Airway surface of (A) WT and (B) STARD7 KO mice surveyed by SEM. Fractures (arrow) were prominent along the airway surface of STARD7 KO mice. Scale bar is $5 \mu \mathrm{m}$.
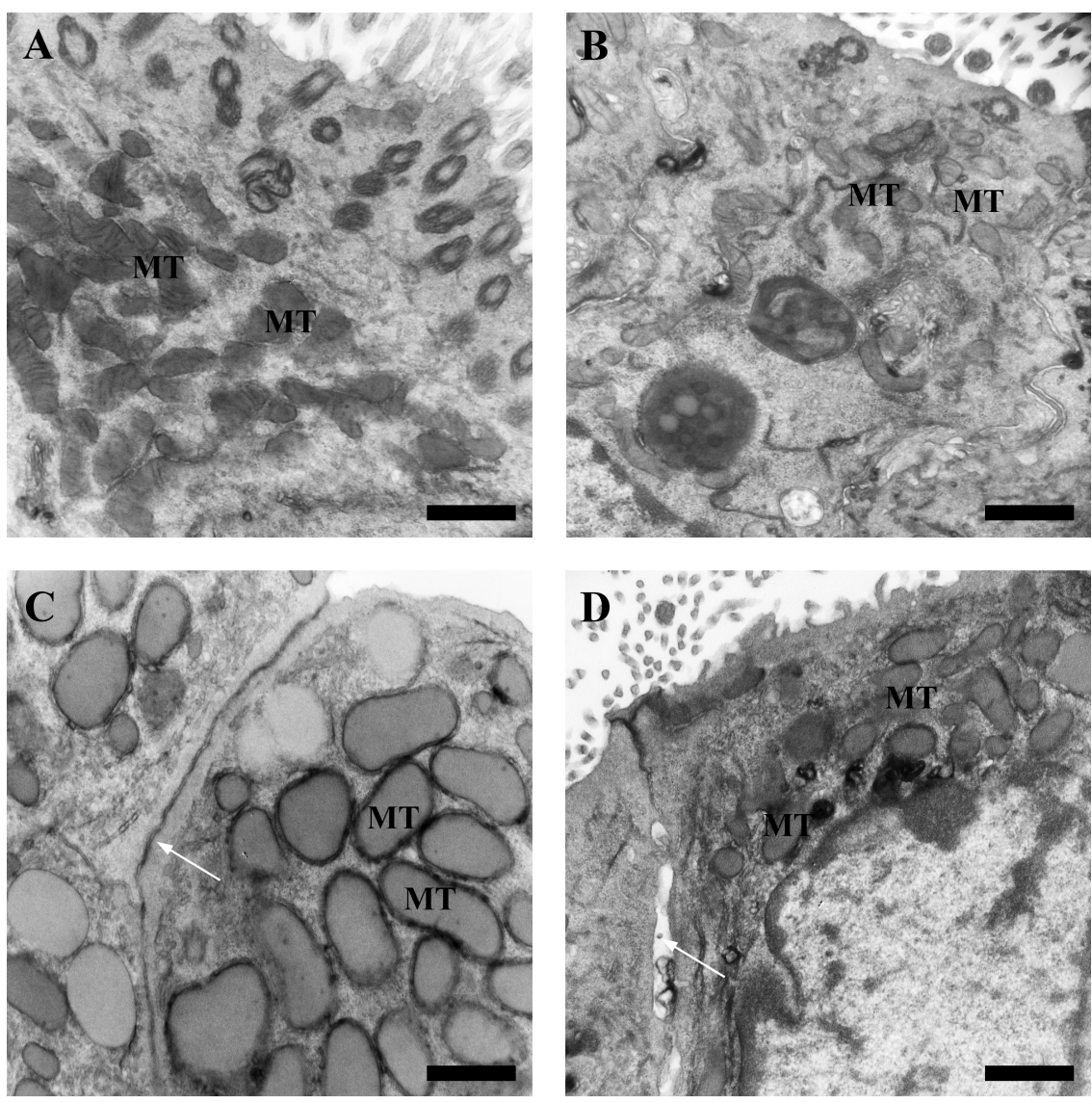

Figure 2. Ciliated bronchiolar epithelial cells and Clara Cells of the WT (A and C) and STARD7 KO mice (B and D). The number and size of mitochondria was significantly reduced in ciliated bronchiolar epithelial cells and Clara cells of the STARD7 KO mice (B and D) compared to WT (A and C). Lipid accumulation was pronounced and integrity of cell junctions (arrow) was compromised in airway epithelial cells of KO mice (B and D). MT: mitochondria. Scale bar is $1 \mu \mathrm{m}$. 